\title{
Secretaries and Administrative Assistants
}

National Cancer Institute

\section{Source}

National Cancer Institute. Secretaries and Administrative Assistants. NCI Thesaurus.

Code C122508.

Secretaries and administrative assistants perform routine clerical and administrative duties. They organize files, draft messages, schedule appointments, and support other staff. 